\title{
DISTRIBUIÇÃO ESPACIAL DE COMPOSTOS ORGÂNICOS DE ESTANHO EM SEDIMENTOS COSTEIROS E EM Phallusia nigra DA BAÍA DE TODOS OS SANTOS E LITORAL NORTE DA BAHIA - BRASIL
}

\author{
Adelmo Lowe Pletsch* \\ Departamento de Biologia e Tecnologia, Universidade Estadual do Norte do Paraná, BR 369, km 54, 86360-000 Bandeirantes - PR, Brasil \\ Magda Beretta \\ Escola Politécnica, Universidade Federal da Bahia, Rua Aristides Novis, 2, 40210-630 Salvador - BA, Brasil \\ Tania Mascarenhas Tavares \\ Instituto de Química, Universidade Federal da Bahia, Rua Barão de Geremoabo, 147, Campus de Ondina, 40170-290 Salvador-BA, Brasil
}

Recebido em 12/12/08; aceito em 16/7/09; publicado na web em 5/2/10

\begin{abstract}
SPATIAL DISTRIBUTION OF ORGANIC TIN COMPOUNDS IN COASTAL SEDIMENT AND Phallusia nigra OF THE TODOS OS SANTOS BAY AND NORTHERN COAST OF BAHIA - BRAZIL. Organic tin compounds were investigated in the sediment of twenty-four stations located in the Todos os Santos Bay and the north coast of Bahia - Brazil. The concentration of these organic tin was also determined in a ascidium species of Phallusia nigra. The speciation and quantification was performed by GC-MS/MS. The highest concentrations were: tributyltin (TBT) $438 \mathrm{ng} \mathrm{g}^{-1}$, dibutyltin (DBT) $207 \mathrm{ng} \mathrm{g}^{-1}$, monobutyltin (MBT) $423 \mathrm{ng} \mathrm{g}^{-1}$, diphenyltin (DPT) $25.8 \mathrm{ng} \mathrm{g}^{-1}$ and monophenyltin (MPT) $55.0 \mathrm{ng} \mathrm{g}^{-1}$. DBT and MBT were found in Phallusia nigra, with highest concentrations of 294.1 and $148.5 \mathrm{ng} \mathrm{g}^{-1}$, respectively.
\end{abstract}

Keywords: organotin; marine sediment; Phallusia nigra.

\section{INTRODUÇÃO}

Os compostos orgânicos de estanho (OTs) incluem-se entre as classes de poluentes mais abundantes em ambientes estuarinos, resultantes de atividades portuárias bem como de descargas industriais e urbanas. O estudo dos efeitos destes compostos lipofílicos nos mecanismos de biotransformação de espécies que vivem em contato estreito com o sedimento reveste-se de grande importância na compreensão ecotoxicológica, pois normalmente estas espécies são neurotóxicas e prováveis interferentes endócrinos, mesmo em níveis muito baixos. Conhecer o estado de oxidação e alquilação é importante para compreender a relação de toxicidade, mobilidade e biodisponibilidade. ${ }^{1}$

Os compostos orgânicos de estanho, que têm como função eliminar a incrustação de animais que produzem rugosidade nas embarcações, apresentam toxicidade em organismos aquáticos alvos e não alvos. Os principais compostos utilizados em pinturas de embarcações são o tributilestanho (TBT) e o trifenilestanho (TPT). Para os butílicos a toxicidade aumenta com o número de radicais butil de um até três, depois é reduzida com a adição de um quarto radical. Por isso, o estudo desta classe de compostos deve incluir além do cátion dissociado $\left(\mathrm{Bu}_{3} \mathrm{Sn}^{+}\right)$, seus metabólitos presumivelmente formados pela desbutilação progressiva até o $\mathrm{Sn}$ inorgânico $-\mathrm{Bu}_{3} \mathrm{Sn}^{+} \rightarrow \mathrm{Bu}_{2} \mathrm{Sn}^{2+} \rightarrow \mathrm{BuSn}^{3+}$ $\rightarrow \mathrm{Sn}^{4+} .{ }^{2}$ Os compostos fenílicos de estanho são mais tóxicos ao meio ambiente do que os butílicos, e o trifenilestanho (TPT) se decompõe de forma semelhante a difenilestanho (DPT) e monofenilestanho (MPT).

A relação entre as concentrações das diversas espécies dos compostos orgânicos de estanho no sedimento pode fornecer informações temporais sobre o grau de contaminação e apontar fontes de introdução destes compostos no ambiente. ${ }^{3}$ A predominância do tributilestanho (TBT) sobre as concentrações dos compostos dibutil estanho (DBT) e monobutilestanho (MBT) indica um aporte recente via anti-incrustrante do TBT no ambiente aquático. Relações entre

*e-mail: adelmolowe@gmail.com
TBT/DBT > 1,0 nos sedimentos superficiais caracterizam uma fonte local de TBT para a coluna d'água. ${ }^{4}$

A especiação dos compostos orgânicos de estanho (OTs) é extremamente importante para se compreender a relação de toxicidade, mobilidade e biodisponibilidade, ${ }^{1}$ no entanto, o desenvolvimento de técnicas analíticas para a determinação de espécies orgânicas de metais em amostras ambientais não é trivial devido aos problemas relacionados à amostragem, extração e quantificação final. Os compostos orgânicos de estanho apresentam-se no meio ambiente em concentrações extremamente baixas, desta maneira, torna-se necessário o desenvolvimento de métodos analíticos cada vez mais seletivos, precisos e sensíveis. ${ }^{5}$ Esse problema é encontrado na maioria dos métodos que não atendem às necessidades ambientais, solicitando várias etapas de preparo de amostra como pré-concentração e adição de padrão para que se possa quantificar de modo confiável o composto de interesse.

Na especiação dos compostos orgânicos de estanho a cromatografia a gás (GC) possui inúmeras vantagens, dentre elas, elevada resolução, alta eficiência na introdução de amostra, baixo ruído de fundo devido à elevada pureza do gás inerte empregado como fase móvel e possibilidade de emprego de padrão interno. As desvantagens típicas do emprego da GC para especiação de compostos orgânicos de estanho estão no fato desses se apresentarem na forma moderadamente iônica, de baixa estabilidade térmica e alta reatividade, sendo necessário realizar uma etapa de derivação anterior à separação e determinação, o que pode afetar a exatidão e a precisão, particularmente para análise de especiação de amostras complexas, tais como as ambientais e biológicas. ${ }^{6} \mathrm{O}$ limite de detecção para o TBT e seus derivados no sedimento e biota para essa técnica tem sido na faixa de 0,5 e $5,0 \mathrm{ug} \mathrm{kg}^{-1}$, respectivamente. $^{7} \mathrm{~A}$ cromatografia a gás - espectrometria de massa (GC-EM-EM) apresenta alta resolução na separação dos componentes de interesse da amostra, com grande sensibilidade e seletividade. Esse sistema de detecção é um dos mais versáteis e vem sendo frequentemente usado paralelamente com outros métodos de detecção de compostos orgânicos de estanho para controle de qualidade dos resultados obtidos e foi a utilizada no presente trabalho. ${ }^{8-10}$ 
O objetivo deste estudo foi avaliar a presença de diversas espécies de compostos orgânicos de estanho (OTs): tributilestanho (TBT), dibutilestanho (DBT), monobutilestanho (MBT), difenilestanho (DPT) e monofenilestanho (MPT) nos sedimentos superficiais (2 cm superiores) de 24 estações de amostragem e na espécie Phallusia nigra (ascídia), bem como identificar as possíveis fontes de introdução destes no ambiente marinho.

A escolha da Baía de Todos os Santos (BTS) e o litoral norte (LN) para esse estudo se deve ao fato de, além de ser a maior baía do Brasil, apresentar diversas fontes potenciais de compostos orgânicos de estanho, como grande atividade portuária, com terminais de carga e descarga geral (com containers) de óleo, produtos diversos e passageiros, bem como diversos estaleiros onde são fabricadas desde lanchas utilizadas para recreio, transporte de passageiros e pesca, até embarcações de grande porte, utilizadas em águas nacionais e internacionais. No litoral norte são encontrados pequenos portos e os efluentes de emissários submarinos do esgotamento sanitário de Salvador, da central de tratamento do Pólo Petroquímico de Camaçari e de uma indústria de óxido de titânio. No entanto, os impactos esperados são menores do que os da BTS, pois a área costeira do Atlântico é de grande energia, com dinâmica de águas intensa e, portanto, grande renovação de água e sedimento. ${ }^{11}$

A espécie Phallusia nigra foi escolhida para este estudo devido a sua frequente ocorrência em regiões com atividades portuárias, em que outras espécies de interesse estão geralmente ausentes e, mesmo com poucas informações sobre a acumulação e os possíveis efeitos dos compostos orgânicos de estanho sobre a espécie, seu estudo se faz interessante. Além de dados sobre acumulação de vanádio, um grande número de compostos químicos, especialmente os mais abundantes em regiões poluídas próximas a portos, como arsênio, cádmio, cromo, cobre, ferro, chumbo, mercúrio, selênio e estanho como tributilestanho, podem ser tolerados e acumulados por ascídias adultas. ${ }^{12,13}$ Todos esses compostos, mas especialmente o TBT, têm desenvolvido efeitos tóxicos sobre embriões e larvas que estão expostas. ${ }^{14} \mathrm{O}$ TBT tem atuado também como um desregulador sobre muitas enzimas em ascídias. ${ }^{15,16}$

Na Baía de Todos os Santos já foram realizados vários estudos determinando níveis de metais pesados, agrotóxicos e compostos de origem petrogênica, envolvendo espécies comestíveis, como moluscos e peixes, entre outras. ${ }^{17-21}$ Porém, não existem estudos sobre a acumulação de compostos orgânicos de estanho, sendo o presente trabalho pioneiro no Brasil sobre a Phallussia nigra para estes compostos.

\section{PARTE EXPERIMENTAL}

\section{Coleta das amostras}

As amostragens de sedimento superficial (aproximadamente 2 cm superiores) foram realizadas em 24 estações na Baía de Todos os Santos e no litoral norte da Bahia (Figura 1S, material suplementar). Foram também coletadas amostras de Phallusia nigra em 6 destas, entre maio de 2003 e abril de 2004, Tabela 1. Os sedimentos superficiais e os animais foram coletados em locais que atendem alguns critérios básicos: pontos localizados próximos a fontes potenciais de orgânicos de estanho, como portos, estaleiros e marinas, mas não tão próximos a ponto de ocorrer contaminação direta de partículas de tinta provenientes de raspagem de cascos presentes nos sedimentos superficiais, que poderia influenciar diretamente nos resultados; pontos que poderão servir de background para avaliar a contaminação por atividades antrópicas sobre os demais locais; pontos com grande fluxo de embarcações e outras atividades como emissários de esgotos; a espécie Phallusia Nigra foi coletada nos mesmos locais do sedimento quando encontrada.
As amostras de sedimento foram coletadas nos pontos E15 (Madre de Deus), E16 (Rio Vermelho) e E17 (Rio Jaguaribe) com o uso de draga Shipek e nos demais pontos com tubos cilíndricos de alumínio, com $5,0 \mathrm{~cm}$ diâmetro por $60 \mathrm{~cm}$ de comprimento, efetuadas por mergulhadores profissionais, treinados para coletas em ambientes marinhos. As amostras foram armazenadas em embalagens descartáveis de alumínio para compostos orgânicos e recipientes de plástico para compostos inorgânicos, previamente descontaminados e colocados em caixas de isopor contendo gelo seco e transportados ao laboratório.

A biota foi coletada em recipientes de alumínio, contendo água do local e transportados até o laboratório, onde a água foi drenada e a Phallusia nigra imediatamente removida do casco de proteção, secada e preparada para análise.

Tabela 1. Localização dos pontos de coleta de sedimento e biota na Baía de Todos os Santos e litoral norte

\begin{tabular}{|c|c|c|c|c|}
\hline Estação & Local & Latitude & Longitude & $\begin{array}{c}\text { Profundidade } \\
\text { da água (m) }\end{array}$ \\
\hline E1* & $\begin{array}{l}\text { B. Naval Aratu } 1 \\
\text { (Dique seco) }\end{array}$ & $12^{\circ} 47^{\prime} .58 \mathrm{~S}$ & $38^{\circ} 30^{\prime} .07 \mathrm{~W}$ & 7,5 \\
\hline E2 & $\begin{array}{l}\text { B. Naval Aratu } 2 \\
\text { (Cais Bravo) }\end{array}$ & $12^{\circ} 47^{\prime} .48 \mathrm{~S}$ & $38^{\circ} 29^{\prime} .64 \mathrm{~W}$ & 9,0 \\
\hline E3 & $\begin{array}{l}\text { B. Naval Aratu } 3 \\
\text { (Entrada da Baia) }\end{array}$ & $12^{\circ} 47^{\prime} \cdot 30 \mathrm{~s}$ & $38^{\circ} 29^{\prime} .97 \mathrm{~W}$ & 9,5 \\
\hline E4 & Baia Marina & $12^{\circ} 58^{\prime} 37.4 \mathrm{~S}$ & $38^{\circ} 31^{\prime} 13.4 \mathrm{~W}$ & 6,5 \\
\hline E5* & $\begin{array}{l}\text { Bom Despacho1 } \\
\quad(\text { Ferry Boat })\end{array}$ & $12^{\circ} 55^{\prime} 31.2 \mathrm{~S}$ & $38^{\circ} 37^{\prime} 08.6 \mathrm{~W}$ & 4,7 \\
\hline E6 & Bom Despacho2 & $12^{\circ} 55^{\prime} 23.5 \mathrm{~S}$ & $38^{\circ} 37^{\prime} 07.1 \mathrm{~W}$ & 7,2 \\
\hline E7 & Bom Despacho3 & $12^{\circ} 55^{\prime} 30.4 \mathrm{~S}$ & $38^{\circ} 37^{\prime} 12.3 \mathrm{~W}$ & 5,0 \\
\hline E8* & $\begin{array}{l}\text { Centro Náutico } \\
\text { (Cidade Baixa) }\end{array}$ & $12^{\circ} 58^{\prime} 23.0 \mathrm{~S}$ & $38^{\circ} 30^{\prime} 57.2 \mathrm{~W}$ & 4,5 \\
\hline E9* & Ilha do Medo & $12^{\circ} 51^{\prime} 40.1 \mathrm{~S}$ & $38^{\circ} 42^{\prime} 31.4 \mathrm{~W}$ & 2,0 \\
\hline E10 & Ilha dos Frades & $12^{\circ} 45^{\prime} 53.0 \mathrm{~S}$ & $38^{\circ} 38^{\prime} 37.0 \mathrm{~W}$ & 4,0 \\
\hline E11* & Porto/Salvador & $12^{\circ} 57^{\prime} 25.4 \mathrm{~S}$ & $38^{\circ} 30^{\prime} 28.8 \mathrm{~W}$ & 14,4 \\
\hline E12* & $\begin{array}{l}\text { T. São Joaquim } \\
\text { (Ferry Boat) }\end{array}$ & $12^{\circ} 57^{\prime} 05.8 \mathrm{~S}$ & $38^{\circ} 30^{\prime} 12.2 \mathrm{~W}$ & 6,5 \\
\hline E13 & T. Mar Grande & $12^{\circ} 57^{\prime} 47.5 \mathrm{~S}$ & $38^{\circ} 36^{\prime} 19.9 \mathrm{~W}$ & 2,2 \\
\hline E14 & Porto da Ribeira & $12^{\circ} 71^{\prime} 02.0 \mathrm{~S}$ & $38^{\circ} 30^{\prime} 30.0 \mathrm{~W}$ & 4,0 \\
\hline E15 & Madre de Deus & $05^{\circ} 41^{\prime} 01.1 \mathrm{~S}$ & $85^{\circ} 95^{\prime} 030 \mathrm{~W}$ & 0,0 \\
\hline E16 & Rio Vermelho & $13^{\circ} 01^{\prime} 50.6 \mathrm{~S}$ & $38^{\circ} 28^{\prime} 52.4 \mathrm{~W}$ & 22,0 \\
\hline E17 & Rio Jaguaribe & $12^{\circ} 58^{\prime} 50.6 \mathrm{~S}$ & $38^{\circ} 22^{\prime} 44.3 \mathrm{~W}$ & 24,0 \\
\hline E18 & $\begin{array}{l}\text { Praia do Forte } \\
\text { (Embarcações) }\end{array}$ & $12^{\circ} 34^{\prime} 72.5 \mathrm{~S}$ & $38^{\circ} 00^{\prime} 17.2 \mathrm{~W}$ & 4,0 \\
\hline E19 & $\begin{array}{l}\text { Praia do Forte } \\
\text { (Litoral Norte) }\end{array}$ & $12^{\circ} 32^{\prime} 22.4 \mathrm{~S}$ & $37^{\circ} 58^{\prime} 55.6 \mathrm{~W}$ & 8,0 \\
\hline E20 & $\begin{array}{l}\text { Arembepe } \\
\text { (Porto) }\end{array}$ & $12^{\circ} 46^{\prime} 45.8 \mathrm{~S}$ & $38^{\circ} 10^{\prime} 62.8 \mathrm{~W}$ & 4,0 \\
\hline E21 & $\begin{array}{c}\text { Cetrel } \\
\text { (Emissário) }\end{array}$ & $12^{\circ} 44^{\prime} 55.1 \mathrm{~S}$ & $38^{\circ} 08^{\prime} 96.8 \mathrm{~W}$ & 9,0 \\
\hline E22 & $\begin{array}{l}\text { Milenium } \\
\text { (Emissário) }\end{array}$ & $12^{\circ} 48^{\prime} 93.3 \mathrm{~S}$ & $38^{\circ} 12^{\prime} 61.1 \mathrm{~W}$ & 8,0 \\
\hline E23 & Jardim de Allah & $13^{\circ} 00^{\prime} 05.8 \mathrm{~S}$ & $38^{\circ} 25^{\prime} 92.8 \mathrm{~W}$ & 24,0 \\
\hline E24 & Pituba & $13^{\circ} 00^{\prime} 86.0 \mathrm{~S}$ & $38^{\circ} 27^{\prime} 54.2 \mathrm{~W}$ & 10,5 \\
\hline
\end{tabular}

Nestas estações foram coletadas espécies de biota (Phallusia nigra). 


\section{Preparação das amostras e procedimentos de extração e derivação}

As amostras de sedimento e biota foram secas em liofilizador a $-55^{\circ} \mathrm{C} \operatorname{logo}$ após a chegada ao laboratório e acondicionadas em frascos de vidro, previamente descontaminados. A fração abaixo de 120 mesh dos sedimentos foi selecionada por peneiração, utilizando-se peneira de metal para análise de compostos orgânicos e peneira de nylon para análise de metais. Todas as amostras foram armazenadas a $-18^{\circ} \mathrm{C}$ até ser realizada análise química.

Para o tratamento prévio das amostras utilizou-se a metodologia de Abalos et al. e Schubert et al.. ${ }^{22,23}$ Essa metodologia foi primeiramente utilizada por Godoi ${ }^{24}$ em um trabalho pioneiro no Brasil na análise de compostos OTs. As duas primeiras modificações descritas a seguir foram anteriormente testadas e utilizadas por Fernandez e a terceira, foi implementada neste estudo: ${ }^{25}$ (1) a derivação foi realizada em atmosfera inerte de $\mathrm{N}_{2}$; (2) visto que a reação é altamente exotérmica, a água e o $\mathrm{HCl}$ concentrado eram mantidos sob refrigeração, para provocar somente destruição do excesso de Grignard e do precipitado formado, evitando a degradação dos compostos OTs; (3) o reagente de derivação $\left(\mathrm{PeMgBr}, 2 \mathrm{~mol} \mathrm{~L}^{-1}\right.$ em dietiléter) foi armazenado em um isopor sob refrigeração a $0^{\circ} \mathrm{C}$ até a utilização, o que facilitou o processo de transferência para o frasco de derivação e reduziu sua degradação.

Cerca de $2 \mathrm{~g}$ de cada amostra liofilizada foram transferidas para um tubo de centrífuga com tampa forrada de teflon e recobertas com lâmina de alumínio, evitando-se assim decomposição fotoquímica. Adicionaram-se $192 \mathrm{ng}$ de triciclo-hexilestanho (TCvT) como rastreador da recuperação dos analitos (surrogate), deixando-se em contato por $12 \mathrm{~h}$ com a matriz, para atingir o equilíbrio. ${ }^{26} \mathrm{~A}$ extração foi efetuada com com $10 \mathrm{~mL}$ de toluenoácido acético (10:40) com sonicação por $5 \mathrm{~min}$, em presença de cobre ativado para eliminação do enxofre e sulfeto. ${ }^{23}$ A separação do solvente extrator contendo os analitos foi obtida com auxílio de centrifugação a $2000 \mathrm{rpm}$ por $3 \mathrm{~min}$, sendo o sobrenadante transferido para um funil de separação. O processo foi repetido duas vezes, e as três porções de sobrenadante combinadas no mesmo recipiente. A fim de eliminar o ácido acético e aumentar a solubilidade dos OTs mono e dissubstituídos na fase orgânica, uma nova extração foi feita com $10 \mathrm{~mL}$ de uma solução aquosa de APDC $0,05 \%$, sendo a fase aquosa descartada, o resíduo de água removido por passagem por uma coluna contendo $2,0 \mathrm{~g}$ de $\mathrm{Na}_{2} \mathrm{SO}_{4}$ ativado. Esse procedimento foi repetido duas vezes. O extrato foi evaporado a vácuo até $2,0 \mathrm{~mL}$, seguindo-se a etapa de derivação por pentilação.

Para a derivação utilizou-se $2,5 \mathrm{~mL}$ de reagente de Grignard (brometo de n-pentilmagnésio) $2 \mathrm{M}$ em dietiléter adicionado ao extrato concentrado, com agitação por $1 \mathrm{~min}$. O excesso de reagente de Grignard foi eliminado com a adição de $12 \mathrm{~mL}$ de água Milli-Q e algumas gotas de $\mathrm{HCl}$ (25\%), mantendo-se o recipiente em banho de gelo, uma vez que a reação é exotérmica. O extrato derivado foi recuperado com extração em triplicada com porções de 5,00 mL de hexano e, em seguida, reduzido a $2 \mathrm{~mL}$ com evaporação em rotavapor. Os lipídios e outros possíveis interferentes foram removidos por cromatografia líquida em coluna contendo $3,0 \mathrm{~g}$ de alumina ativada e 2,0 $\mathrm{g}$ de $\mathrm{Na}_{2} \mathrm{SO}_{4}$ ativado utilizando $12 \mathrm{~mL}$ de hexano como eluente. $\mathrm{O}$ extrato foi reduzido em rotavapor, posteriormente avolumado para 4,00 mL quando se acrescentou como padrão interno o tetrabutiestanho (TeBT) para a determinação posterior.

A eficiência da derivação foi avaliada em duplicata para um pool de padrões de compostos OTs e a metodologia foi validada usando material de referência certificado (MRC) para sedimento de portos, PACs-2, da National Research Council do Canadá (Ottawa on Canadá) certificado para TBT e DBT.

\section{Determinação de compostos orgânicos de estanho em sedimento e Phallusia nigra}

As análises dos compostos OTs foram realizadas em duplicata em um cromatógrafo a gás, modelo 3600 da Varian, acoplado a dois espectrômetros de massa sequenciados do tipo ín trap com amostrador automático. A coluna capilar utilizada foi uma VF-5 (5\% fenil/95\% metil silicone) - Varian, com $30 \mathrm{~m}$ de comprimento e 0,25 $\mathrm{mm}$ de diâmetro interno e espessura do filme de $0,25 \mu \mathrm{m}$. As injeções de $2 \mu \mathrm{L}$ foram conduzidas com sandwich (intercalação de ar antes e depois da amostra ainda na seringa de injeção)-(0,5 $\mu \mathrm{L}$ ar $-2 \mu \mathrm{L}$ de amostra $-0,5 \mu \mathrm{L}$ de ar) com uma programação de temperatura da coluna de $\mathrm{T}_{\mathrm{i}} 60^{\circ} \mathrm{C}$, de $60^{\circ} \mathrm{C}$ numa taxa de $8{ }^{\circ} \mathrm{C} \mathrm{min}{ }^{-1}$ até $280{ }^{\circ} \mathrm{C}$, sendo mantida nessa temperatura por $5 \mathrm{~min}$. $\mathrm{O}$ gás de arraste utilizado foi hélio, com fluxo de $1,1 \mathrm{~mL} \mathrm{~min}^{-1}$. Injetor: $\mathrm{T}_{\mathrm{i}} 150{ }^{\circ} \mathrm{C}$, taxa de aquecimento de $300{ }^{\circ} \mathrm{C}$ até $\mathrm{T}_{\mathrm{f}} 250{ }^{\circ} \mathrm{C}$. Os dados dos espectros de massas foram adquiridos no modo SIM (monitoramento do íon selecionado, de melhor sensibilidade), sendo monitorados os íons primários e secundários dos orgânicos de estanho.

Os compostos OTs encontrados nas amostras de sedimento e biota foram quantificados através da relação de áreas com um padrão interno, o TeBT (tetrabutilestanho - 147,03 ng mL-1), que não necessita de derivação, levando-se em conta o fator de resposta calculado para cada composto.

\section{Análise de estanho total}

A digestão das amostras foi feita com $\mathrm{HNO}_{3}$ e peróxido de hidrogênio 30\%, a quente e analisada por ICP-MS (Élan 6000, PerkinElmer-Sciex), utilizando-se um nebulizador do tipo cross flow, com câmara de nebulização do tipo Rayton para introdução da amostra.

\section{Análises de parâmetros auxiliares}

O potencial hidrogeniônico foi determinado imediatamente após a chegada das amostras no laboratório. O método utilizado estima a atividade dos íons $\mathrm{H}^{+}$na suspensão do sedimento, utilizando $\mathrm{CaCl}_{2}$ $10^{-2} \mathrm{~mol} \mathrm{~L}^{-1}$ para a manutenção do equilíbrio iônico da solução. Foram transferidos $8 \mathrm{~cm}^{3}$ de sedimento para um béquer de poliuretano de 60 $\mathrm{mL}$ e adicionados $20 \mathrm{~mL}$ de $\mathrm{CaCl}_{2} 10^{-2} \mathrm{~mol} \mathrm{~L}^{-1}$. A solução foi agitada durante $15 \mathrm{~min}$ a $250 \mathrm{rpm}$ e a leitura foi efetuada utilizando-se um potenciômetro marca Marte, modelo MB-10, após 30 min. ${ }^{27}$

As amostras de sedimento foram submetidas à análise granulométrica para determinação das frações areia (grossa e fina), silte e argila. As análises foram realizadas de acordo com o método desenvolvido pela EMBRAPA ${ }^{28}$ nos laboratórios de solo do Departamento Nacional de Obras Contra a Seca (DNOCS).

As determinações do carbono orgânico (CO) e enxofre (S) foram realizadas pelo método de combustão a seco (analisador elementar da CE Instruments, modelo EA 1110). As medidas foram feitas em duplicata, usando-se cerca de 2 a $5 \mathrm{mg}$ de sedimento seco (homogeneizado). A descarbonatação foi efetuada com ácido clorídrico 1 mol L ${ }^{-1}$, controlando-se o $\mathrm{pH}$ (em torno de 2,0) para evitar perdas da fração mais solúvel da matéria orgânica. ${ }^{29}$

Os teores de lipídios foram determinados por soxhlet, usando-se etér de petróleo como extrator.

\section{RESULTADOS E DISCUSSÃO}

\section{Compostos orgânicos de estanho em sedimentos}

A exatidão do procedimento analítico dos compostos orgânicos de estanho foi verificada com material de referência certificado, re- 
Tabela 2. Valores encontrados para TBT, DBT, MBT e $\mathrm{Sn}_{\text {total }}$ expressos em ng g ${ }^{-1}$ sedimento (peso seco) no padrão certificado PACs-2

\begin{tabular}{lcccc}
\hline Compostos & TBT & DBT & MBT & \multicolumn{1}{c}{$\mathrm{Sn}_{\text {total }}$} \\
\hline Valor determinado $(\mathrm{N}=3)$ & $979,4 \pm 50,9$ & $1125 \pm 110,6$ & $348,8 \pm 58,9$ & $20000 \pm 1000^{\mathrm{a}}$ \\
Valor certificado & $980,0 \pm 130,0$ & $1090 \pm 150,0$ & $300^{\mathrm{b}}$ & $19800 \pm 2500$ \\
\hline
\end{tabular}

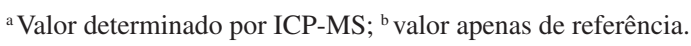

Tabela 3. Medidas de $\mathrm{pH}$, concentrações de carbono orgânico e enxofre em mg g-1 $\mathrm{gBT}^{-1}$, DBT, MBT, MPT e DPT como Sn, em ng g-1 em sedimento (peso seco), determinadas por CG-MS-MS

\begin{tabular}{|c|c|c|c|c|c|c|c|c|c|c|}
\hline Estação & $\mathrm{pH}$ & $\mathrm{CO}$ & S & Argila (\%) & TBT & DBT & MBT & MPT & DPT & ¿OTs \\
\hline E1 & 8,05 & 12,5 & 2.92 & 85 & 158 & 207 & 423 & 55,0 & 25,8 & 870 \\
\hline E2 & 8,22 & 7,74 & 0.20 & 57 & 127 & 46,5 & 67,8 & $<1,95$ & $<1,93$ & 242 \\
\hline E3 & 7,94 & 5,72 & 1.17 & 52 & 64,2 & 73,9 & 80,2 & $<1,95$ & 17,4 & 235 \\
\hline E4 & 8,44 & 1,24 & 0.86 & 32 & 53,5 & $<2,70$ & $<3,15$ & $<1,95$ & $<1,93$ & 53,5 \\
\hline E5 & 8,08 & 7,73 & $<\mathrm{ld}$ & 54 & $<2,53$ & $<2,70$ & 67,3 & $<1,95$ & $<1,93$ & 67,3 \\
\hline E6 & 8,18 & 1,83 & $<\mathrm{ld}$ & 46 & nd & nd & nd & nd & nd & nd \\
\hline E7 & 7,98 & 5,72 & 0.10 & 43 & nd & nd & nd & nd & nd & nd \\
\hline E8 & 8,55 & $<\mathrm{Id}$ & $<\mathrm{ld}$ & 33 & $<2,53$ & $<2,70$ & $<3,15$ & $<1,95$ & $<1,93$ & $<\mathrm{ld}$ \\
\hline E9 & 8,03 & 1,34 & $<\mathrm{ld}$ & 23 & $<2,53$ & 22,0 & $<3,15$ & $<1,95$ & $<1,93$ & 22,0 \\
\hline E10 & 7,97 & 0,76 & 0.11 & 16 & $<2,53$ & $<2,70$ & 132 & $<1,95$ & $<1,93$ & 132 \\
\hline E11 & 8,29 & 10,9 & $<\mathrm{ld}$ & 34 & 438 & 45,1 & 134 & $<1,95$ & $<1,93$ & 618 \\
\hline E12 & 8,23 & 2,48 & 1.60 & 39 & 26,5 & $<2,70$ & $<3,15$ & $<1,95$ & $<1,93$ & 26,5 \\
\hline E13 & 8,28 & 2,17 & 1.04 & 36 & $<2,53$ & $<2,70$ & $<3,15$ & $<1,95$ & $<1,93$ & $<$ ld \\
\hline E14 & 7,98 & 7,39 & 7.33 & 83 & $<2,53$ & $<2,70$ & 205 & $<1,95$ & $<1,93$ & 205 \\
\hline E15 & 6,90 & 1,73 & 0.39 & 46 & $<2,53$ & $<2,70$ & $<3,15$ & $<1,95$ & $<1,93$ & $<$ ld \\
\hline E16 & 8,14 & 2,85 & 1.01 & 3 & 88,6 & $<2,70$ & $<3,15$ & $<1,95$ & $<1,93$ & 88,6 \\
\hline E17 & 8,19 & 2,79 & $<1 d$ & 5 & 45,5 & $<2,70$ & $<3,15$ & $<1,95$ & $<1,93$ & 45,5 \\
\hline E18 & 8,09 & 3,90 & 2.91 & 27 & $<2,53$ & 42,5 & $<3,15$ & $<1,95$ & $<1,93$ & 42,5 \\
\hline E19 & 8,40 & 0,95 & 0.21 & 9 & $<2,53$ & $<2,70$ & $<3,15$ & $<1,95$ & $<1,93$ & $<\mathrm{ld}$ \\
\hline E20 & 8,37 & 1,54 & $<\mathrm{ld}$ & 26 & $<2,53$ & $<2,70$ & $<3,15$ & $<1,95$ & $<1,93$ & $<1 d$ \\
\hline E21 & 8,48 & 0,63 & $<\mathrm{ld}$ & 24 & $<2,53$ & $<2,70$ & $<3,15$ & $<1,95$ & $<1,93$ & $<1 d$ \\
\hline E22 & 8,35 & 1,23 & $<\mathrm{ld}$ & 23 & $<2,53$ & $<2,70$ & $<3,15$ & $<1,95$ & $<1,93$ & $<\mathrm{ld}$ \\
\hline E23 & 8,37 & 1,93 & $<1 d$ & 2 & 49,4 & $<2,70$ & $<3,15$ & $<1,95$ & $<1,93$ & 49,4 \\
\hline E24 & 8,36 & 1,25 & $<\mathrm{ld}$ & 2 & $<2,53$ & $<2,70$ & $<3,15$ & $<1,95$ & $<1,93$ & $<\mathrm{ld}$ \\
\hline
\end{tabular}

nd = não determinado; $1 \mathrm{~d}=$ limite de detecção

sultando em $99 \%$ para TBT e $103 \%$ para DBT e podem ser vistos na Tabela 2. Para o composto MBT, para o qual o valor da amostra não é certificado e apenas de referência, a exatidão foi de $84 \%$.

O surrogate triciclo-hexilestanho (TCyT), adicionado no início de cada procedimento para corrigir para recuperações incompletas, mostrou recuperações entre 76,8 e $113 \%$ nas amostras de sedimento e entre 52,2 e 89,0\% na Phalussia nigra, sendo que, para a maioria das amostras foi sempre acima de $85 \%$.

Os limites de detecção dos compostos OTs nos sedimentos e biota (massa seca) foram em $\mathrm{ng} \mathrm{g}^{-1}$ : TBT 2,53; DBT 2,70; MBT 3,15; DPT 1,93; MPT 1,95; TCyT 4,33. O desvio padrão para os compostos OTs estudados variou entre 0,020 a 2,6 ng g $^{-1}$.

Os resultados da especiação dos compostos orgânicos de estanho para sedimentos superficiais da Baía de Todos os Santos e litoral norte mostram uma grande variabilidade presencial e de concentração dentro da ampla avaliação espacial.

Dentre os fenílicos, foram estudados somente o difenilestanho (DPT), encontrado nas estações E1 (25,8 $\left.\mathrm{ng} \mathrm{g}^{-1}\right)$ e E3 (17,4 $\left.\mathrm{ng} \mathrm{g}^{-1}\right)$ e o monofenilestanho (MPT) na estação E1 (55,0 $\left.\mathrm{ng} \mathrm{g}^{-1}\right)$. Ambos os fenílicos foram encontrados dentro da Baía de Aratu, sendo, provavelmente, provenientes de atividades marítimas no local, como nos estaleiros da Base Naval de Aratu, onde são realizadas recuperações nos cascos de navios envolvendo raspagens e pinturas.

As concentrações dos butílicos e fenílicos em sedimentos são apresentadas na Tabela 3, juntamente com o somatório dos compostos orgânicos de estanho e parâmetros como pH, carbono orgânico, enxofre e granulometria.

Os butílicos, TBT, DBT e MBT foram encontrados simultaneamente nas estações E1, E2, E3, e E11; já nas demais estações a distribuição foi consoante, com as concentrações do TBT variando de 26,5 a 438 ng g $^{-1}$.

Quando ocorre predominância de TBT em relação a DBT e MBT, isso indica aporte recente via anti-incrustrante na coluna d'água. ${ }^{3} \mathrm{~A}$ relação TBT/DBT >1,0 caracteriza, para sedimentos superficiais, uma fonte local de TBT para a coluna d'água. ${ }^{30}$ Essa relação foi superada somente em 4 estações; dentre essas, é possível apontar fontes locais nas estações E2 e E11 com predominância do composto TBT sobre os demais butílicos. Essa relação deve ser avaliada com muito cuidado, 
pois as estações de amostragem são muito próximas e a região tem velocidades de correntes muito baixas (até $20 \mathrm{~cm} / \mathrm{s}$ ). ${ }^{31}$ As relações encontradas para este estudo deveriam apontar fonte local de TBT também na estação E1, localizada próxima a uma fonte de emissão, em um dique seco onde são feitas reformas de embarcações; no entanto, essa divergência pode ser compreendida pelas condições hidrodinâmicas deste local, pois esse dique é cheio com água do mar para a alocação da embarcação que será submetida à reforma e logo em seguida esvaziado sob forte pressão, quando a água é "empurrada" para fora provocando alta ressuspensão dos sedimentos, favorecendo assim a degradação do TBT a DBT bem como facilitando o seu arraste para regiões mais distantes.

Já nas demais estações a origem dos compostos DBT e MBT pode estar associada a fatores como a fotólise direta na coluna d'água, determinante no processo de degradação, visto que a degradação do TBT é considerada um processo rápido na coluna d'água, apresentando meias-vidas características da ordem de dias. ${ }^{32}$ Essa desbutilação abiótica do TBT na coluna d'água pode ser esperada, pois a água da BTS apresenta elevada transparência e baixa profundidade (próximo de 6 m). ${ }^{33}$ No entanto, os compostos DBT e MBT encontrados em muitas estações, possivelmente, não estão associados somente à degradação na coluna d'água, mas também à decomposição química do grupo TBT na etapa de hidrólise da tinta do tipo copolímero aplicado nos cascos dos navios, ${ }^{34}$ onde o biocida é lixiviado ao invés da dissolução da partícula da tinta. No Brasil, atualmente são utilizadas tintas do tipo copolímero na pintura das embarcações. ${ }^{35}$ Portanto, a presença dos compostos DBT e MBT em estações como E9 e E10, que estão afastadas de potenciais fontes antrópicas pode ser esperada, visto que durante o transporte destes compostos ao longo da baía as reações são cineticamente favorecidas com temperaturas mais elevadas e alta incidência solar. A distribuição dos compostos OTs também exibe correlações positivas com os teores de matéria orgânica, justificando a variabilidade presencial em diversos estudos. ${ }^{36}$

O somatório dos compostos orgânicos de estanho destaca altas concentrações nas estações localizadas dentro da Baía de Aratu $\left\{\sum\right.$ OTs $\left(\mathrm{ng} \mathrm{g}^{-1}\right)(\mathrm{E} 1=870),(\mathrm{E} 2=242)$ e $\left.(\mathrm{E} 3=235)\right\}$ quando comparado aos teores encontrados nas demais estações. Isso pode ser explicado pelo fato da região apresentar intenso fluxo de navios cargueiros, emissários de indústrias químicas (fábrica de insumos químicos), indústrias portuárias, siderurgias, estaleiros, marinas e uma base naval (próxima dos pontos de coleta) onde são recuperados cascos de embarcações envolvendo raspagens e pinturas, que favorecem a contaminação do ambiente aquático. Também foi encontrado um alto teor no Porto de Salvador ( $\sum \mathrm{OTs}=618 \mathrm{ng} \mathrm{g}^{-1}$ ), onde ocorre grande tráfego de embarcações e uma atividade portuária intensa.

O potencial hidrogênionico exerce influência importante na disponibilidade dos compostos orgânicos de estanho no sedimento. ${ }^{37}$ Estudos já realizados com peixes e organismos presentes em sedimento também mostraram que a biodisponibilidade é mais alta para um $\mathrm{pH}$ mais elevado, ${ }^{38}$ e com maior afinidade do TBT pelo sedimento quando comparado ao DBT, com sequência de hidrofobicidade TBT $>$ DBT. ${ }^{39}$ Nas estações estudadas na BTS e LN o pH do sedimento é próximo de 8 , condição favorável para disponibilização dos compostos OTs para a biota.

A matéria orgânica (MO), constituída em proporção definida de carbono e podendo ser calculada com base no teor desse elemento presente nas amostras (\% MO =\% x 1,725), é também uma variável importante para se compreender as concentrações e a disponibilidade dos compostos orgânicos de estanho no sedimento. Estudos mostram que em altas concentrações de $\mathrm{MO}$ a bioconcentração decresce de 2-98\%. ${ }^{40,41}$ Para os resultados de $\mathrm{CO}$ encontrados nos pontos estudados na BTS e LN são obtidos altos teores nas estações E1, E2, E5, E11 e E14. Os resultados mostram alta concordância com o composto
TBT justificando, assim, as altas concentrações nestas estações. No entanto, é esperada uma baixa disponibilidade do TBT nestas estações em função do alto teor de MO, que complexa fortemente esses compostos. $\mathrm{O}$ enxofre que também estabelece fortes interações com organometálicos no sedimento foi encontrado em teor elevado na estação E14. Os teores de argila não mostraram significância estatística na interpretação dos resultados.

Para critérios de qualidade ambiental implementados em vários países europeus, os valores para TBT em sedimentos estão entre 1-2 $\mu \mathrm{g} \mathrm{kg}^{-1} \cdot{ }^{42} \mathrm{De}$ acordo com este critério, os níveis do composto TBT encontrados nos sedimentos estudados na BTS e LN, quando detectados, apresentaram-se acima de tal critério de classificação para se inferir a qualidade ambiental.

De acordo com a classificação apresentada por Waite ${ }^{43}$ para o TBT, áreas levemente contaminadas apresentam concentrações que variam entre $<10$ e $50 \mathrm{ng} \mathrm{g}^{-1}$ (como Sn); áreas consideradas moderadamente contaminadas, próximas de amarras estão entre 60 e 200; áreas consideradas altamente contaminadas estão entre 300 e 1000. Com base nessa classificação os sedimentos das estações E4, E12, E17 e E23 encontram-se classificados como levemente contaminados. As estações E1, E2, E3 e E16 podem ser classificadas como moderadamente contaminadas. Nestes locais existem intensas atividades antrópicas geradoras do composto TBT, como cais marítimos, onde são realizadas reformas de embarcações e pinturas dos cascos de navios. Já a estação E11 é classificada como altamente contaminada, tendo-se em vista que neste local há um intenso trafego de embarcações de médio e grande porte, juntamente com movimentação de contêineres e outras atividades.

As concentrações dos compostos butílicos da Baía de Todos os Santos são semelhantes às obtidas em sedimentos superficiais de outros países. ${ }^{44,45}$ Em estudos realizados na Baía de Guanabara, Brasil, foram encontradas altas concentrações de compostos butílicos de estanho em áreas de atracação para pequenas embarcações, concluindo-se que estas atividades representam fontes significativas de contaminação destes compostos para o ambiente marinho. ${ }^{35}$ Conclusões semelhantes foram descritas em trabalho desenvolvido no litoral de São Paulo. ${ }^{46}$ Felizzola ${ }^{47}$ realizou um estudo no sedimento superficial da BTS e encontrou valores bem inferiores aos deste estudo realizado na mesma região. Essa diferença pode ser justificada, visto que as estações de coleta deste trabalho estão localizadas mais próximas a locais onde as atividades antrópicas são mais intensas, como portos e marinas, diferentemente dos pontos de coleta considerados no trabalho de Fellizola, que se localizavam em áreas não tão próximas de fontes antrópicas de compostos OTs.

\section{Compostos orgânicos de estanho em Phallusia nigra}

As ascídias são filtradores e removem o plâncton da corrente hídrica que passa pela faringe. A corrente hídrica é produzida pelo batimento dos cílios laterais nas margens das fendas branquiais, filtrando uma enorme quantidade de água para sua alimentação. Um espécime de ascídia preta (com alguns centímetros de comprimento) pode passar $173 \mathrm{~L}$ de água através de seu corpo em $24 \mathrm{~h}$. Nas ascídias as células morulares concentram os metais pesados em cachos intracelulares de vesículas semelhantes a bagas. A Phallusia nigra é um tunicado bentônico séssil, possuindo um número reduzido de predadores, o que sugere a presença de eficiente defesa química. Estudos realizados em laboratório têm demonstrado que ascídias expostas ao composto TBT o acumulam. ${ }^{48}$

Apesar de ser muito comum na costa brasileira, a espécie Phallusia nigra esteve ausente na maioria das estações da BTS e LN, por isso foi estudado em apenas 6 das 24 estações. Ainda, em 2 estações a quantidade de indivíduos encontrados foi muito pequena, sendo então 
Tabela 4. Concentrações dos compostos TBT, DBT e MBT, como Sn, em ng g ${ }^{-1}$ na biota (Phallusia nigra) (peso seco), determinados por CG-MS-MS e Sn ${ }_{\text {total }}$ em $\mu \mathrm{g} \mathrm{g} \mathrm{g}^{-1}$, por ICP-MS

\begin{tabular}{|c|c|c|c|c|c|c|c|}
\hline Estação & Local & Lipídios\% & $\mathrm{Sn}_{\text {total }}$ & TBT & DBT & MBT & $\sum$ OTs \\
\hline E1 & B. Naval Aratu 1 (Dique seco) & 4,62 & 7,47 & $<\mathrm{LD}$ & 158,9 & 140,5 & 299,4 \\
\hline E5 & Bom Despacho1 (Ferry Boat) & 4,76 & 11,7 & $<\mathrm{LD}$ & 225,2 & 148,5 & 373,7 \\
\hline E8 & Centro Náutico (Cidade Baixa) & nd & 25,5 & nd & nd & nd & nd \\
\hline pE9 & Ilha do Medo & nd & 1,57 & nd & nd & nd & nd \\
\hline E11 & Porto/Salvador & 4,48 & 25,8 & $<\mathrm{LD}$ & 159,2 & 122,2 & 281,4 \\
\hline E12 & T. São Joaquim (Ferry Boat) & 4,76 & 16,3 & $<\mathrm{LD}$ & 294,1 & 141,7 & 435,8 \\
\hline
\end{tabular}

$\mathrm{nd}=$ não determinado. $\mathrm{LD}=$ Limite de detecção

determinada somente a concentração de estanho total nas amostras das estações E8 e E9.

O estanho metálico total foi avaliado na espécie Phallusia nigra das 6 estações, sendo encontrado em todas as amostras com concentrações variando entre 1,57 e $25,8 \mu \mathrm{g} \mathrm{g}^{-1}$ em massa seca. A menor concentração foi encontrada na espécie coletada na Ilha do Medo e a maior no porto de Salvador, conforme apresentado na Tabela 4.

Dos compostos OTs estudados na espécies foram encontrados somente os butílicos DBT e MBT detectados em altas concentrações. O composto TBT não foi encontrado em nenhuma das amostras; provavelmente este composto pode estar sofrendo rápida degradação na água e, como estas espécies são filtradoras, podem ter acumulado os desbutilados do TBT. É observado ainda que o DBT é encontrado em teor superior ao MBT. Para explicar essa maior concentração dos derivados do TBT, estudos mais detalhados precisam ser realizados com a água, o material particulado e as espécies nestes locais, pois também existe a possibilidade de ocorrer a degradação após a assimilação destes pela ascídia preta. No entanto, essa última suposição é pouco provável porque vários estudos já comprovaram que o TBT acumulado por outras espécies de biota apresenta baixa taxa de degradação. ${ }^{49}$

Comparando-se os teores dos desbutilados DBT e MBT na biota com os encontrados no sedimento, das estações E1 e E5, nota-se uma elevada relação. Isso se deve, provavelmente, ao fato destes locais apresentarem uma elevada movimentação de embarcações favorecendo a ressuspensão do TBT para a coluna d'água e a sua degradação. Essa mesma relação já não ocorre nas outras duas estações onde a biota foi estudada, possivelmente, pela diferença no comportamento hidrodinâmico do ambiente.

Os teores de lipídios em peso seco foram $\mathrm{E} 1=4,62$; E5= 4,76; $\mathrm{E} 11=4,48 ; \mathrm{E} 12=4,76$. As correlações entre o somatório dos OTs com os teores de lipídios foram elevadas ( $\mathrm{r}=0,88486$ e $\mathrm{p}=0,01151)$, mostrando que esses compostos podem estar sendo retidos devido à alta solubilidade em compostos de baixa polaridade conferida aos lipídios.

\section{CONCLUSÕES}

De modo geral, o estudo mostrou que os compostos orgânicos de estanho apresentaram distribuição diversificada, sendo encontrados no sedimento de $50 \%$ das estações da BTS e LN. Em algumas estações de coleta o composto TBT foi encontrado em concentrações superiores ao DBT, indicando que as introduções destes compostos no ambiente aquático foram recentes, exceto nas estações E1 e E3 onde as relações foram opostas, com predominância do composto DBT, indicando que as incorporações ocorrem a longo prazo. Os compostos fenílicos de estanho foram encontrados apenas nas amostras da Baía de Aratu, sugerindo que as intensas atividades navais realizadas na região possam ser as fontes de introdução. Os níveis de compostos orgânicos de estanho nos sedimentos da Baía de Todos os Santos foram mais altos do que no litoral norte da Bahia onde a dinâmica de águas é intensa, com grande renovação de água e sedimento. A presença do DBT e MBT, produtos da degradação do TBT, na Phallusia nigra indica a presença destes na coluna d'água.

\section{MATERIAL SUPLEMENTAR}

A Figura 1S apresenta a localização dos pontos de coleta distribuídos ao longo da Baía de Todos os Santos e litoral norte da Bahia.

Está disponível gratuitamente em http://quimicanova.sbq.org.br, na forma de aquivo PDF, com acesso livre.

\section{AGRADECIMENTOS}

À CAPES pela bolsa de estudo. Ao Laboratório de Química Analítica Ambiental - LAQUAM - do Departamento de Química Analítica da UFBA pela infraestrutura e suporte técnico proporcionados para a execução deste trabalho. À Marinha do Brasil - Base Naval de Aratu - pelo apoio nas coletas de sedimentos na Baía de Aratu. Ao Prof. Dr. R. Carreira, da Universidade Estadual do Rio de Janeiro, pelo auxílio nas análises de carbono orgânico e ao Prof. Dr. N. Miekeley, PUC-RJ, pela análise de metais.

\section{REFERÊNCIAS}

1. Muñoz, J.; Baena, J. R.; Gallego, M.; Valcárcel, M.; J. Chromatogr., A 2004, 1023, 175.

2. Bryan, G. W.; Langston, W. J.; Environ. Pollut. 1992, 76, 89.

3. Kuballa, J.; Wilken, R. D.; Jantzen, E.; Kwan, K. K.; Chau, Y. K.; Analyst 1995, 120, 667.

4. Stuer-Lauridsen, F.; Dahl, B.; Chemosphere 1995, 30, 831.

5. Carlier-Pinasseau, C.; Astruc, A.; Lespes, G.; Astruc, M.; J. Chromatogr, A 1996, 750, 317.

6. Abalos, M.; Bayona, J. M.; Compaño, R.; Granados, M.; Leal, C.; J. Chromatogr. 1997, 788, 1.

7. World Health Organization International - WHO; Tributyiltin Compounds, Environmental Health Criteria: Geneva, 1990.

8. Masucci, J. A.; Caldwell, G. W. Em Modern Practice of Gas Chromatography, Grob, R. L.; Barry, E.F., eds.; $4^{\text {th }}$ ed.; John Wiley \& Sons: New York, 2004 .

9. Venkatesan, M. I.; Chalaux, N.; Bayona, J. M.; Zeng, E.; Environ. Pollut. 1998, 99, 263.

10. Tolosa, I.; Merlini, L.; De Bertrand, N.; Bayona, J. M.; Albaigés, J.; Environ. Toxicol. Chem. 1992, 11, 145.

11. Pletsch, A. L.; Tese de Doutorado, Universidade Federal da Bahia, Brasil, 2006.

12. Papadopoulou, C.; Kanias, G. D.; Mar. Pollut. Bull. 1977, 8, 229.

13. Lambert, G.; Can. J. Zool. 2005, 83, 34.

14. Ten Hallers-Tjabbes, C. C.; Wegener, J. W.; Hattum, V. B.; Kemp, J. F.; Ten Hallers, E.; Reitsema, T. J.; Boon J. P.; Mar. Environ. Res. 2003, 55, 203. 
15. Bellas, J.; Vázquez, E.; Beiras, R.; Water Res. 2001, 35, 2905.

16. Cima, F.; Ballarin, L.; Bressa, G.; Martinucci, G.; Burighel, P.; Ecotoxicol. Environ. Saf. 1996, 35, 174.

17. Oliveira, F. M.; Tavares, T. M.; Beretta, M.; Analytica 2005,14, 45.

18. Tavares, T. M.; Rocha, V.C.; Porte, C.; Barceló, D.; Albaigés, J.; Mar. Pollut. Bull. 1988, 19, 575.

19. Porte, C. ; Barceló, D. ; Tavares, T. M.; Rocha, V. C.; Albaigés, J. ; Arch. Environ. Contam. Toxicol. 1990, 19, 263.

20. Tavares, T. M. ; Beretta, M.; Costa, M. C.; Chemosphere 1999, 38, 1445.

21. Machado, J. C. V.; Tavares, T. M; Proceedings of the World Water Congress of the International Water Association (IWA) 2000, 3.

22. Abalos, M.; Bayona, J. M.; Quevauviller, P.; Appl. Organomet. Chem. 1998, $12,1$.

23. Schubert, P.; Fernández-Escobar, I.; Rosenberg, E.; Bayona, J. M.; J. Chromatogr., A 1998, 810, 245.

24. Godoi, A. F. L.; Favoreto, R.; Santiago-Silva, M.; J. Chromatogr. 2003, $58,97$.

25. Fernandez, M.; Wagener, A. de L. R.; Limaverde, A. M.; Scofield, A. de L.; Pinheiro, F. M.; Rodrigues, E.; Mar. Environ. Res. 2005, 59, 435.

26. Quevauviller, P.; Morabito, R.; Anal. Chem. 2000, 19, 86.

27. Pavan, M. A.; Bloch, M. F.; Zempulski, H. C.; Miyazawa, M.; Zocoler, D. C.; Manual de Análise Química de Solo e Controle de Qualidade, $1^{\mathrm{a}}$ ed., IAPAR: Londrina, 1992.

28. EMBRAPA; Manual de métodos de análise do solo, $2^{\mathrm{a}}$ ed., EMBRAPA: Rio de Janeiro, 1997.

29. Froelich, P. N.; Limnol. Oceanogr. 1980, 25, 564.

30. Stuer-Lauridsen, F.; Dahl, B.; Chemosphere 1995, 30, 831.

31. Lessa, G. C.; Dominguez, J. M. L.; Bittencourt, A. C. S. P.; Brichta, A.; An. Acad. Bras. Cienc. 2001, 73, 245.

32. Clark, E. L.; Sterrit, R. M.; Lester, J. N.; Environ. Sci. Technol. 1988, 22,600 .
33. Centro de Recursos Ambientais da Bahia - CRA; Diagnóstico da Concentração de Metais Pesados e Hidrocarbonetos de Petróleo nos Sedimentos e Biota da Baía de Todos os Santos, Salvador, 2004, vol. II.

34. Champ, M. A.; Lowestein, F. L.; Ocean. Fall. 1987, 30, 69.

35. Fernandez, M. A. S.; Tese de Doutorado, Pontifícia Universidade Católica do Rio de Janeiro, Brasil, 2001.

36. Buggy, C. I.; Tobin, J. M.; Environ. Pollut. 2006, 143, 294.

37. Rüdel, H.; Ecotoxicol. Environ. Saf. 2003, 56, 180.

38. Fent, K.; Toxicology 1996, 26, 1.

39. Hoch, M.; Appl. Geochem. 2001, 16, 719.

40. Haitzer, M.; Höss, S.; Traunspurger, W.; Steinberg, C.; Chemosphere 1998, 37, 1335 .

41. Fent, K.; Looser, P.; Water Res. 1995, 29, 1631.

42. Ceulemans, M.; Slaets, S.; Adams, F. C.; Talanta 1998, 46, 395.

43. Waite, M. E.; Waldock, M. J.; Thain, J. E.; Smith, D. J.; Milton, S. M.; Mar. Environ. Res. 1991, 32, 89.

44. Suzuki, T.; Kondo, K.; Uchiyama, M.; Murayama, M.; J. Agric. Food Chem. 1999, 47, 3886.

45. Biselli, S.; Bester, K.; Huhnerfuss, H.; Fent, K.; Mar. Pollut. Bull. 2000, $40,233$.

46. Godoi, A. F. L.; Montone, R. C.; Silva, M. S.; J. Chromatogr. 2003, 15, 985.

47. Felizzola, F. J.; Wagener, A. L. R.; Almeida, A. C.; Lin, W.O.; Quim. Nova 2008, 31, 89.

48. Radford, J. L.; Hutchinson, A. E.; Burandt, M.; Raftos, D. A.; J. Invert. Pathol. 2000, 76, 242.

49. Seligman, P. F.; Valkirs, A. O.; Stang, P. M.; Lee, R. F.; Mar. Pollut. Bull. 1988, 19, 531. 


\section{DISTRIBUIÇÃO ESPACIAL DE COMPOSTOS ORGÂNICOS DE ESTANHO EM SEDIMENTOS COSTEIROS E EM Phallusia nigra DA BAÍA DE TODOS OS SANTOS E LITORAL NORTE DA BAHIA - BRASIL}

\section{Adelmo Lowe Pletsch*}

Departamento de Biologia e Tecnologia, Universidade Estadual do Norte do Paraná, BR 369, km 54, 86360-000 Bandeirantes - PR, Brasil Magda Beretta

Escola Politécnica, Universidade Federal da Bahia, Rua Aristides Novis, 2, 40210-630 Salvador - BA, Brasil

Tania Mascarenhas Tavares

Instituto de Química, Universidade Federal da Bahia, Rua Barão de Geremoabo, 147, Campus de Ondina, 40170-290 Salvador - BA, Brasil

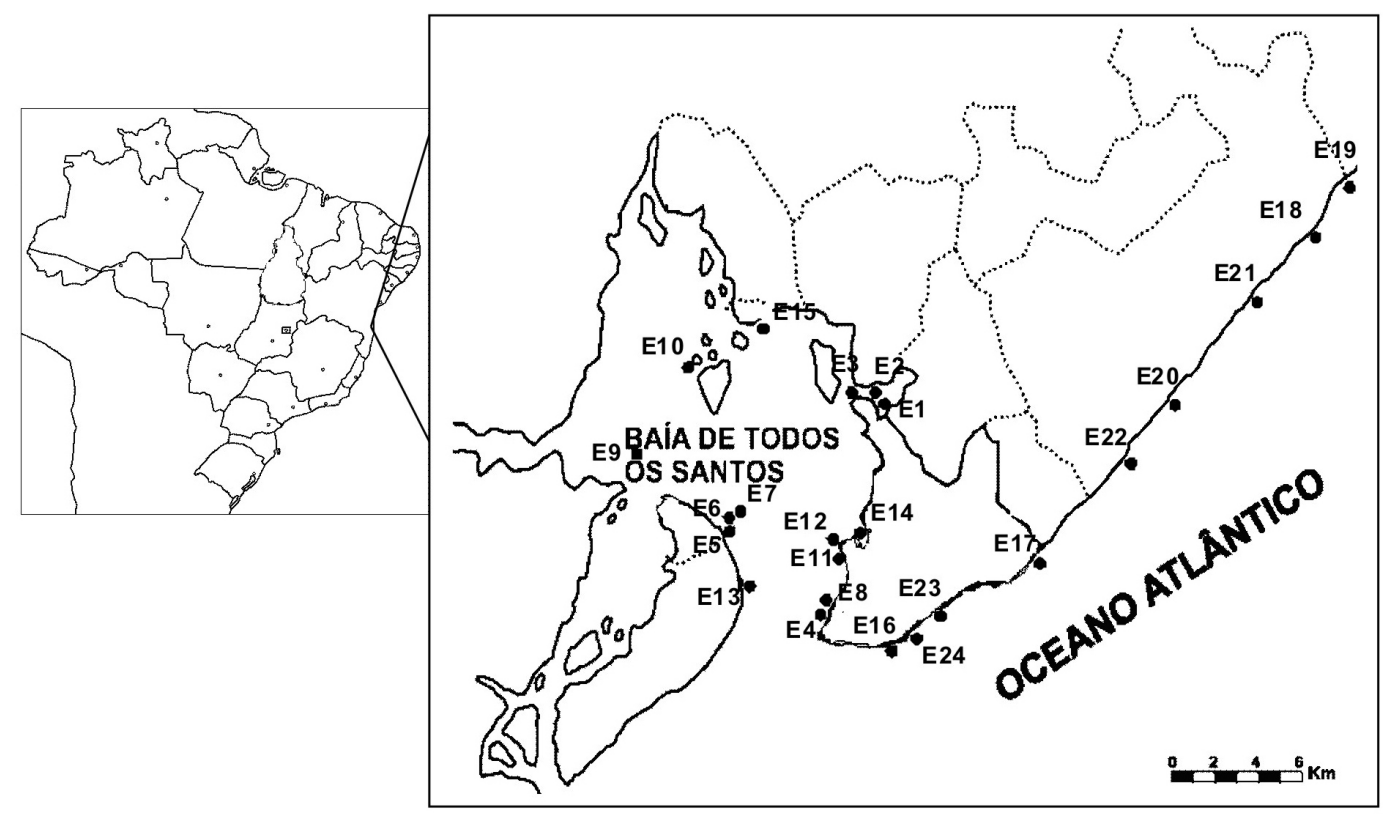

Figuras 1S. Mapa das estações de coleta na BTS e Litoral Norte. 